\title{
Local Degree of Freedom of Clutter for Reduced-Dimension Space-Time Adaptive Processing with MIMO Radar
}

\author{
Yiduo Guo, ${ }^{1,2}$ Jian Gong, ${ }^{1,2}$ and Yu Xiao $\mathbb{D}^{2}$ \\ ${ }^{1}$ National Laboratory of Radar Signal Processing, Xidian University, Xi'an, Shaanxi 710071, China \\ ${ }^{2}$ Air Force Engineering University, Xi'an, Shaanxi 710051, China \\ Correspondence should be addressed to Yu Xiao; yuxiao_afeu@126.com
}

Received 31 July 2017; Revised 18 October 2017; Accepted 28 November 2017; Published 18 March 2018

Academic Editor: Wen-Qin Wang

Copyright (c) 2018 Yiduo Guo et al. This is an open access article distributed under the Creative Commons Attribution License, which permits unrestricted use, distribution, and reproduction in any medium, provided the original work is properly cited.

\begin{abstract}
Degree of freedom (DOF) of clutter in the reduced-dimension (RD) domain, which is called local DOF (LDOF), is of great importance for RD MIMO-STAP (space-time adaptive processing for multiple-input multiple-output radar) algorithms. In this paper, the LDOF equivalence of different RD MIMO-STAP algorithms are firstly proved, and then a generalized LDOF estimation rule under different conditions is developed to estimate the clutter LDOF for MIMO radar effectively. The accuracy of the proposed rule is verified, and how to design RD MIMO-STAP processors under the guidance of the proposed rule is presented through numerical simulations.
\end{abstract}

\section{Introduction}

Airborne radar system explores space-time adaptive processing (STAP) technique to detect slow-moving targets under strong ground clutter $[1,2]$. Originally, the theory of STAP is developed for conventional airborne phased array radar system, which can be considered as a single-input multipleoutput (SIMO) radar system. However, STAP technique for SIMO radar has been well studied, but its capability of clutter suppression needs to be improved. Compared with SIMO radar, multiple-input multiple-output (MIMO) radar enjoys better spatial resolution because of the much larger virtual array than the physical array [3]. Therefore, MIMOSTAP technique has drawn considerable attention focusing on the significant potentials for clutter suppression in recent years $[4,5]$.

According to the principle of STAP theory, the degree of freedom (DOF) of STAP processors should be larger than that of clutter, so the estimation of clutter DOF for SIMO radar and MIMO radar has been intensively studied. For SIMO radar, the well-known Brennan's rule is firstly presented in [6] and then formally proved by Ward in [7]. The extended Brennan's rule to subarray STAP is provided in [8], and DOF estimation in the case of arbitrary arrays is analyzed in [9]. Nonideal radar configurations, such as bistatic arrays and sparse arrays, have been considered in $[10,11]$. For MIMO radar, the extension of Brennan's rule of MIMO radar has been proposed by Chen and Vaidyanathan in [12]; Xie et al. presented the estimation rule for the clutter DOF of the MIMO-phased array radar in [13]; and Li et al. derived the estimation of clutter DOF of wideband MIMO radar in [14]. These references have made great contributions to the successful design of STAP processors.

However, the optimal STAP processor is usually difficult to apply in real-time applications because of the large independent identically distributed (IID) training sample requirement and the expensive computational cost. To solve these problems, many algorithms aiming to reduce the complexity and improve the convergence of STAP in SIMO radar have been proposed. Among them, reduced-dimension (RD) STAP algorithms are of great importance for their simplicity and effectiveness $[15,16]$. For MIMO radar, because of the added dimension created by the orthogonal waveforms, the two problems mentioned above become even more challenging. Fortunately, with slight modifications, many RD STAP algorithms proposed for SIMO radar can also be applied to MIMO radar. With the transformation of RD STAP algorithms, the clutter data are projected onto a lower- 
dimensional subspace; the DOF of clutter in this subspace is called local degree of freedom (LDOF) of clutter. Similar with the full STAP algorithm, the DOF of RD STAP processor would also be larger than the clutter LDOF. In $[17,18]$, Zhang et al. has given the LDOFs estimation formulas of four classical RD STAP algorithms for airborne SIMO radar with uniform linear array and subarray, which play an important role in the development of RD STAP algorithms. Whereas the research on RD MIMO-STAP algorithms is more urgent than that on SIMO-STAP algorithms, the clutter LDOF for airborne MIMO radar is seldom mentioned.

In this paper, we develop the estimation rule of clutter LDOF for airborne MIMO radar with linear array, and the complete proof of the proposed rule is also presented. Although there are $2^{3}$ classes of typical transformation matrix according to the projected domain of the original signal, we only focus on 4 classes of RD MIMO-STAP algorithms, which are extended directly from RD STAP algorithms for SIMO radar named element-space pre-Doppler algorithm, element-space post-Doppler algorithm, beamspace pre-Doppler algorithm, and beam-space pre-Doppler algorithm [7]. This is because these 4 algorithms are usually utilized in practice and the proofs for the other 4 algorithms are actually similar to the previous ones.

The rest of the paper is organized as follows. In Section 2, the clutter model and clutter DOF for airborne MIMO radar is briefly reviewed and the definition of clutter LDOF of MIMO radar is also presented. In Section 3, the conclusion, which indicates that clutter LDOFs of different RD MIMOSTAP algorithms with the same dimension are actually equivalent, is proved. In Section 4, we develop the clutter LDOF estimation rule for airborne MIMO radar under different conditions. In Section 5, numerical simulations are conducted to demonstrate the effectiveness and applications of the proposed clutter LDOF estimation rule. Finally, Section 6 concludes the paper.

\section{Problem Formulation}

2.1. Clutter Model and Clutter DOF. Considering a monostatic side-looking airborne MIMO array radar at the altitude $H$ with the constant moving velocity $V$. As is illustrated in Figure 1, there are $N$ receiving elements uniformly spaced by $d_{R}=\lambda / 2$ and $M$ transmitting elements uniformly spaced by $d_{T}=\gamma d_{R}$, where $\lambda$ is the wavelength and $\gamma$ is the sparse coefficient. The number of pulses at a coherent processing interval (CPI) is $K$ and the pulse repetition frequency (PRF) is $f_{\mathrm{r}}$. The MIMO radar transmits an orthogonal waveform set $\left\{s_{1}, s_{2}, \ldots, s_{M}\right\}$, and these waveforms will be extracted by $M$ matched filters at each receiving element. The clutter is modeled as a Gaussian process.

The $i$ th clutter patch on the $n$th receiving element for the $m$ th waveform and $k$ th pulse can be written as

$$
y_{i}(m, n, k)=\alpha_{i} s_{m} e^{j 2 \pi[\gamma(m-1)+(n-1)] f_{s_{i}}+j 2 \pi(k-1) f_{d_{i}}}
$$

where $\alpha_{i}$ is the reflect coefficient of the $i$ th clutter patch, $f_{s_{i}}=d_{R} \cos \left(\psi_{i}\right) / \lambda$ is the spatial frequency of the $i$ th clutter patch, and $f_{\mathrm{d}_{i}}=2 V \cos \left(\psi_{i}\right) / \lambda f_{r}$ is the Doppler frequency of the ith clutter patch; $\cos \left(\psi_{i}\right)=\cos \left(\theta_{i}\right) \cos (\varphi)$ is the directional cosine.

Since the MIMO radar transmits orthogonal waveforms, the output of the matched filters can be expressed as

$$
\begin{aligned}
c_{i}(m, n, k) & =\alpha_{i} e^{j 2 \pi(n-1) f_{\mathrm{s}_{i}}+j 2 \pi(k-1) f_{\mathrm{d}_{i}}} \sum_{q=1}^{M} s_{m} s_{q}^{H} e^{j 2 \pi \gamma(m-1) f_{\mathrm{s}_{i}}} \\
& =\alpha_{i} e^{j 2 \pi[(n-1)+\beta(k-1)+\gamma(m-1)] f_{s_{i}}},
\end{aligned}
$$

where $\beta=f_{\mathrm{d}_{i}} / f_{\mathrm{s}_{i}}=2 V / d_{R} f_{\mathrm{r}}$.

Therefore, for the $m$ th waveform and $k$ th pulse, the total clutter echoes on the $n$th receiving element can be expressed as

$$
x(m, n, k)=\sum_{i=1}^{N_{\mathrm{c}}} \alpha_{i} e^{j 2 \pi[(n-1)+\beta(k-1)+\gamma(m-1)] f_{\mathrm{s}_{i}}},
$$

where $N_{\mathrm{c}} \gg M N K$ is the number of clutter patches.

Define the temporal steering vector $\mathbf{S}_{\mathrm{T}, i}$, transmitting spatial steering vector $\mathbf{S}_{\mathrm{t}, i}$ and receiving spatial steering vector $\mathbf{S}_{\mathrm{r}, i}$ as

$$
\begin{aligned}
& \mathbf{S}_{\mathrm{T}, i}=\left[1, e^{j 2 \pi \beta f_{s_{i}}}, \ldots, e^{j 2 \pi \beta f_{s_{i}}(K-1)}\right]^{\mathrm{T}} \in C^{K \times 1}, \\
& \mathbf{S}_{\mathrm{t}, i}=\left[1, e^{j 2 \pi \gamma f_{s_{i}}}, \ldots, e^{j 2 \pi \gamma f_{s_{i}}(M-1)}\right]^{\mathrm{T}} \in C^{M \times 1}, \\
& \mathbf{S}_{\mathrm{r}, i}=\left[1, e^{j 2 \pi f_{s_{i}}}, \ldots, e^{j 2 \pi f_{s_{i}}(N-1)}\right]^{\mathrm{T}} \in C^{N \times 1} .
\end{aligned}
$$

The $M N K \times 1$ clutter signal vector, which is obtained by stacking the signals in (3) for $n=1,2, \ldots, N, m=1,2, \ldots, M$, and $k=1,2, \ldots, K$, can be expressed as

$$
\mathbf{x}=\sum_{i=1}^{N_{c}} \alpha_{i} \mathbf{S}_{i}=\mathbf{U} \boldsymbol{\Lambda},
$$

where $\mathbf{S}_{i}=\mathbf{S}_{\mathrm{T}, i} \otimes \mathbf{S}_{\mathrm{t}, i} \otimes \mathbf{S}_{\mathrm{r}, i}$ is the spatial-temporal steering vector; $\mathbf{U}=\left[\mathbf{S}_{1}, \mathbf{S}_{2}, \ldots, \mathbf{S}_{N_{\mathrm{c}}}\right]$ and $\boldsymbol{\Lambda}=\left[\alpha_{1}, \alpha_{2}, \ldots, \alpha_{N_{\mathrm{c}}}\right]^{T}$.

Therefore, the clutter covariance matrix is given as

$$
\mathbf{R}_{x}=E\left[x x^{H}\right]=\mathbf{U} \boldsymbol{\Sigma} \mathbf{U}^{H},
$$

where $\boldsymbol{\Sigma}=\operatorname{diag}\left(\alpha_{1}^{2}, \alpha_{2}^{2}, \ldots, \alpha_{N_{c}}^{2}\right) ; E[\cdot]$ and $(\cdot)^{H}$ denote statistical expectation and the conjugate transpose, respectively.

The clutter DOF is defined as $r=\operatorname{rank}\left(\mathbf{R}_{x}\right)$. Because $\boldsymbol{\Sigma}$ is a positive definite matrix and $\operatorname{span}\left(\mathbf{R}_{x}\right)=\operatorname{span}(\mathbf{U})$, the clutter DOF is equal to $r=\operatorname{rank}(\mathbf{U})$.

Let $\mathbf{f}_{\mathrm{s}}=\left[f_{\mathrm{s} 1}, f_{\mathrm{s} 2}, \ldots, f_{\mathrm{sN}}\right]^{\mathrm{T}}$; $\mathbf{U}$ can be rewritten as $\mathbf{U}=\left[1, \ldots, e^{j 2 \pi[(n-1)+\beta(k-1)+\gamma(m-1)] \mathbf{f}_{\mathrm{s}}}, \ldots, e^{j 2 \pi[(N-1)+\beta(K-1)+\gamma(M-1)] \mathbf{f}_{\mathrm{s}}}\right]$.

If $\beta \leq N$ and $\gamma \leq N+\beta(K-1)$ are both integers, some row vectors in $\mathbf{U}$ will be exactly the same and there will be at most $N+\beta(K-1)+\gamma(M-1)$ distinct row vectors in $\mathbf{U}$. Thus, when $N+\beta(K-1)+\gamma(M-1) \leq M N K$, the clutter $\mathrm{DOF}$, which is equated to the rank of $\mathbf{U}$, is approximately 


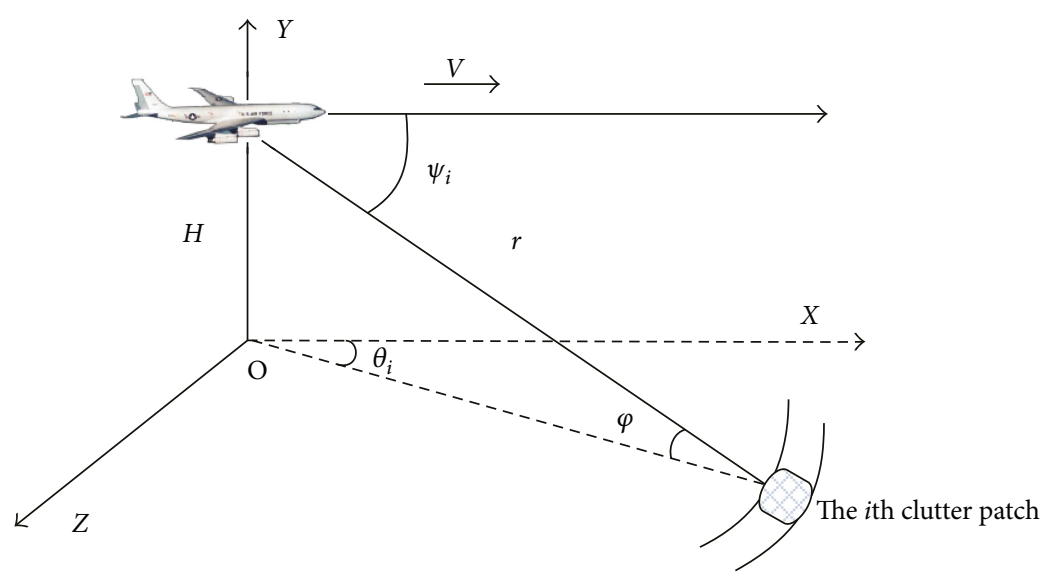

(a) The geometry of carrier aircraft

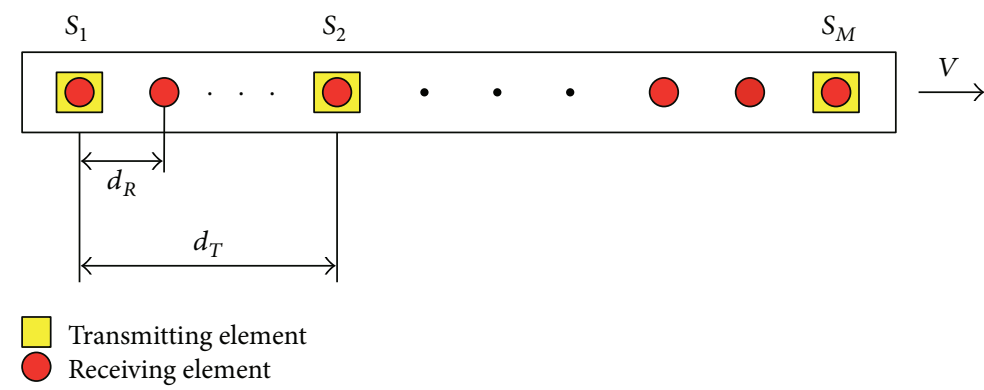

(b) The model of the array and the signal

FIGURE 1: The model of monostatic side-looking airborne MIMO radar.

$$
r=\operatorname{rank}(\mathbf{U}) \approx N+\beta(K-1)+\gamma(M-1) .
$$

Equation (8) is the so-called estimation formula of clutter DOF for airborne MIMO radar provided by Chen et al. in [12], which can be viewed as the extension of Brennan's rule to the MIMO radar case. According to (8), we can see that the clutter DOF increases when $\beta$ goes up.

2.2. RD MIMO-STAP and Clutter LDOF. Generally, RD STAP algorithms apply data-independent transformations to prefilter the received signal and then solve an adaptive filtering operation with the dimension-reduced data. Given the transformation matrix $\mathbf{T}$, the dimension-reduced clutter signal can be expressed as

$$
x_{r}=\mathbf{T}^{H} \mathbf{x}=\sum_{i=1}^{N_{c}} \alpha_{i}\left(\mathbf{T}^{H} \mathbf{S}_{i}\right)=\sum_{i=1}^{N_{c}} \alpha_{i} \mathbf{S}_{i, r}=\mathbf{U}_{r} \boldsymbol{\Lambda},
$$

where $\mathbf{U}_{r}=\left[\mathbf{S}_{1, r}, \mathbf{S}_{2, r}, \ldots, \mathbf{S}_{N_{c}, r}\right]$.

For airborne MIMO radar, similar with the airborne SIMO radar, the transformation matrix $\mathbf{T}$ is commonly defined as

$$
\mathbf{T}=\mathbf{T}_{\mathrm{T}} \otimes \mathbf{T}_{\mathrm{r}} \otimes \mathbf{T}_{\mathrm{t}}
$$

where $\mathbf{T}_{\mathrm{T}} \in C^{K \times P}$ is the time-domain transformation matrix and $\mathbf{T}_{\mathrm{r}} \in C^{N \times Q}$ and $\mathbf{T}_{\mathrm{t}} \in C^{M \times O}$ are the receiving and transmitting space-domain transformation matrices, respectively.
Therefore, the dimension-reduced spatial-temporal steering vector and the dimension-reduced clutter covariance matrix can be written as

$$
\begin{aligned}
\mathbf{S}_{i, r} & =\mathbf{T}^{H} \mathbf{S}_{i}=\left(\mathbf{T}_{\mathrm{T}}^{H} \mathbf{S}_{\mathrm{T}, i}\right) \otimes\left(\mathbf{T}_{\mathrm{r}}^{H} \mathbf{S}_{\mathrm{r}, i}\right) \otimes\left(\mathbf{T}_{\mathrm{t}}^{H} \mathbf{S}_{\mathrm{t}, i}\right), \\
\mathbf{R}_{r} & =E\left[x_{r} x_{r}^{H}\right]=\mathbf{T}^{H} \mathbf{U} \boldsymbol{\Sigma} \mathbf{U}^{H} \mathbf{T}=\mathbf{U}_{r} \boldsymbol{\Sigma} \mathbf{U}_{r}^{H} .
\end{aligned}
$$

Definition 1. One has $\boldsymbol{r}_{r}=\operatorname{rank}\left(\mathbf{R}_{r}\right)=\operatorname{rank}\left(\mathbf{U}_{r}\right)$ as the clutter LDOF for airborne MIMO radar, which demonstrates the DOF of clutter in the dimension-reduced subspace. This is extended directly from the definition of LDOF of airborne SIMO radar proposed by [17].

\section{LDOF Equivalence of Different RD MIMO- STAP Algorithms}

In this section, we will prove that clutter LODFs of different RD MIMO-STAP algorithms with $\mathbf{T}_{\mathrm{T}}, \mathbf{T}_{\mathrm{r}}$, and $\mathbf{T}_{\mathrm{t}}$ of the same size are equivalent. This can significantly reduce the complexity of the formulation and proof of the proposed LDOF estimation rule, which will be presented in Section 4. It should be noted that $\beta$ and $\gamma$ are simplified to be integers in our proofs, and when $\beta$ and $\gamma$ are not integers, the method using Fourier series and significant components proposed by Zhang et al. is also suitable for our situation. The readers are referred to $[17,18]$ for more detailed descriptions.

3.1. Clutter LDOF for Element-Space Pre-Doppler (ES PreDop) RD MIMO-STAP. For ES pre-Dop RD MIMO-STAP, 
the dimension-reduced spatial-temporal steering vector has the following form:

$$
\begin{aligned}
\mathbf{S}_{i, r} & =\mathbf{S}_{\mathrm{T}, i}(a: a+P-1) \otimes \mathbf{S}_{\mathrm{r}, i}(b: b+Q-1) \otimes \mathbf{S}_{\mathrm{t}, i}(c: c+O-1) \\
& =\left[e^{j 2 \pi f_{\mathrm{s}_{i}}((q-1)+\beta(p-1)+\gamma(o-1))}\right], \quad q=b: b+Q-1, \\
p & =a: a+P-1, o=c: c+O-1,
\end{aligned}
$$

where $a, b$, and $c$ are constants for the selections of pulses, receiving elements, and transmitting elements.

Thus, $\mathbf{U}_{r}$ can be expressed as

$$
\begin{aligned}
\mathbf{U}_{r}= & {\left[e^{j 2 \pi \mathbf{f}_{s}((a-1)+\beta(b-1)+\gamma(c-1))}, \ldots,\right.} \\
& \left.e^{j 2 \pi \mathbf{f}_{s}((a+Q-2)+\beta(b+P-2)+\gamma(c+O-2))}\right]^{T} .
\end{aligned}
$$

Since the common coefficient $e^{j 2 \pi \mathrm{f}_{\mathrm{s}}((a-1)+\beta(b-1)+\gamma(c-1))}$ does not have any impact on the rank of $\mathbf{U}_{r}$, we could set $a=b=c=1$, which means that the first $P$ pulses, $Q$ receiving elements, and $O$ transmitting elements are selected for this class of RD MIMO-STAP algorithms. Therefore, according to the same reason of [7], the rank of $\mathbf{U}_{r}$, that is, the clutter LDOF, is the number of distinct row vectors in $\mathbf{U}_{r}$, which is equivalent to the number of distinct elements in the following set:

$$
\left\{(q-1)+\beta(p-1)+\left.\gamma(o-1)\right|_{q=1: Q, p=1: P, o=1: O}\right\} .
$$

3.2. Clutter LDOF for Element-Space Post-Doppler (ES PostDop) RD MIMO-STAP. For ES post-Doppler RD MIMOSTAP, the time-domain transformation matrix can be expressed as

$$
\mathbf{T}_{\mathrm{T}}=\left[T_{\mathrm{T} 1}, \ldots, T_{\mathrm{T} p}, \ldots, T_{\mathrm{T} P}\right] \in C^{K \times P},
$$

where $T_{\mathrm{T} p}=\left[1, \ldots, e^{-j 2 \pi(K-1) d_{p} / K}\right]^{T}, p=1,2, \ldots, P, d_{p} \in[1,2$, $\ldots, K]$, is the $p$ th Doppler bin.

Thus, the reduced-dimension temporal steering vector can be written as

$$
\mathbf{S}_{\mathrm{T}, i, r}=\left(\mathbf{T}_{\mathrm{T}}^{H} \mathbf{S}_{\mathrm{T}, i}\right)=\left[\mathbf{S}_{\mathrm{T}, i, r}(1), \ldots, \mathbf{S}_{\mathrm{T}, i, r}(p), \ldots, \mathbf{S}_{\mathrm{T}, i, r}(P)\right]^{T},
$$

where $\quad \mathbf{S}_{\mathrm{T}, i, r}(p)=\sum_{k=0}^{K-1} e^{j 2 \pi k\left(\beta f_{s_{i}}-d_{p} / K\right)}=\left(1-e^{j 2 \pi \beta f_{s_{i}} K}\right) /(1-$ $\left.e^{j 2 \pi\left(\beta f_{s_{i}}-d_{p} / K\right)}\right)$.

With the selection of the first $Q$ receiving elements and $O$ transmitting elements, the transformed spatial-temporal steering vector can be written as

$$
\begin{aligned}
\mathbf{S}_{i, r} & =\mathbf{S}_{\mathrm{T}, i, r} \otimes \mathbf{S}_{\mathrm{r}, i}(1: Q) \otimes \mathbf{S}_{\mathrm{t}, i}(1: O) \\
& =\left[\frac{\left(1-e^{j 2 \pi \beta f_{s_{i}} K}\right) e^{j 2 \pi f_{s_{i}}((q-1)+\gamma(o-1))}}{1-e^{j 2 \pi\left(\beta f_{s_{i}}-d_{p} / K\right)}}\right]_{q=1: Q, q=1: P, o=1: O} .
\end{aligned}
$$

Ignoring the common coefficient $1-e^{j 2 \pi \beta f_{s_{i}}{ }^{K}}, \mathbf{U}_{r}$ can be simplified as

$$
\mathbf{U}_{r}=\left[\frac{1}{1-e^{j 2 \pi\left(\beta \mathbf{f}_{\mathrm{s}}-d_{1} / K\right)}}, \ldots, \frac{e^{j 2 \pi \mathbf{f}_{\mathrm{s}}((Q-1)+\gamma(O-1))}}{1-e^{j 2 \pi\left(\beta \mathbf{f}_{\mathrm{s}}-d_{P} / K\right)}}\right]^{T}
$$

Given $\Pi=\left[\prod_{p=1}^{P} 1-e^{j 2 \pi\left(\beta f_{s, 1}-d_{p} / K\right)}, \ldots, \prod_{p=1}^{P} 1-e^{j 2 \pi\left(\beta f_{s, N_{c}}-d_{p} / K\right)}\right]^{T}$, the rank of $\mathbf{U}_{r}$ is equivalent to that of the following matrix:

$$
\mathbf{U}_{r}^{*}=\left[\Pi \odot \frac{1}{1-e^{j 2 \pi\left(\beta \mathbf{f}_{\mathrm{s}}-d_{1} / K\right)}}, \ldots, \Pi \odot \frac{e^{j 2 \pi \mathbf{f}_{\mathrm{s}}((\mathrm{Q}-1)+\gamma(O-1))}}{1-e^{j 2 \pi\left(\beta \mathbf{f}_{\mathrm{s}}-d_{P} / K\right)}}\right]^{T},
$$

where $\odot$ denotes Hardmard product.

The $[p(Q O-1)+q(O-1)+o]$ th row of $\mathbf{U}_{r}^{*}$ has the form of

$$
\left[e^{j 2 \pi \mathbf{f}_{\mathrm{s}}((q-1)+\gamma(o-1))} \odot\left(h_{p, 0}+h_{p, 1} e^{j 2 \pi \beta \mathbf{f}_{\mathrm{s}}}+\cdots+h_{p, P-1} e^{j 2 \pi \beta \mathbf{f}_{\mathrm{s}}(P-1)}\right)\right]^{T} .
$$

We can see from (20) that every row of $\mathbf{U}_{r}^{*}$ is the linear combination of monomials.

$$
\begin{aligned}
& \exp \left[j 2 \pi \mathbf{f}_{s}((q-1)+\beta(p-1)+\gamma(o-1))\right], \quad q=1 \\
& \quad: Q, p=1: P, o=1: O .
\end{aligned}
$$

The proof in [18] indicates that the rank of $\mathbf{U}_{r}^{*}$, that is, the clutter LDOF, is equivalent to the number of different expressions of (21). Therefore, it is actually the same as the clutter LDOF of the ES pre-Dop RD MIMO-STAP.

3.3. Clutter LDOF for Beam-Space Pre-Doppler (BS Pre-Dop) $R D M I M O-S T A P$. In this case, the receiving and transmitting space-domain transformation matrices can be expressed as

$$
\begin{aligned}
& \mathbf{T}_{r}=\left[T_{r 1}, \ldots, T_{r q}, \ldots, T_{r Q}\right] \in C^{N \times Q}, \\
& \mathbf{T}_{t}=\left[T_{t 1}, \ldots, T_{\mathrm{to}}, \ldots, T_{\mathrm{tO}}\right] \in C^{M \times O},
\end{aligned}
$$

where $\quad T_{r q}=\left[1, \ldots, e^{-j 2 \pi(N-1) b_{r q} / N}\right]^{T}, \quad q=1,2, \ldots, Q$, $b_{r q} \in[1,2, \ldots, Q]$, is the $q$ th selected receiving beam; $T_{\text {to }}=\left[1, \cdots, e^{-j 2 \pi(M-1) b_{t, o} / M}\right]^{T}, \quad o=1,2, \ldots, O, \quad b_{\text {to }} \in[1,2, \ldots$, $O]$, is the oth selected transmitting beam.

So the dimension-reduced receiving and transmitting spatial steering vector can be expressed as

$$
\begin{aligned}
& \mathbf{S}_{\mathrm{r}, i, r}=\left[\mathbf{S}_{\mathrm{r}, i, r}(1), \ldots, \mathbf{S}_{\mathrm{r}, i, r}(q), \ldots, \mathbf{S}_{\mathrm{r}, i, r}(Q)\right]^{T}, \\
& \mathbf{S}_{\mathrm{t}, i, r}=\left[\mathbf{S}_{\mathrm{t}, i, r}(1), \ldots, \mathbf{S}_{\mathrm{t}, i, r}(o), \ldots, \mathbf{S}_{\mathrm{t}, i, r}(O)\right]^{T},
\end{aligned}
$$

where $\mathbf{S}_{\mathrm{r}, i, r}(q)=\sum_{n=0}^{N-1} e^{j 2 \pi n\left(f_{s_{i}}-b_{r q} / N\right)}=\left(1-e^{j 2 \pi f_{s_{i}} N}\right) /\left(1-e^{j 2 \pi\left(f_{s_{i}}-b_{r q} / N\right)}\right)$ and $S_{\mathrm{t}, i, r}(o)=\sum_{m=0}^{M-1} e^{j 2 \pi m\left(f_{s_{i}}-b_{\mathrm{to}} / M\right)}=\left(1-e^{j 2 \pi f_{s_{i}} M}\right) /\left(1-e^{j 2 \pi\left(f_{s_{i}}-b_{\mathrm{to}} / M\right)}\right)$.

Selecting the first $P$ pulses, the transformed spatialtemporal steering vector can be written as

$$
\mathbf{S}_{i, r}=\mathbf{S}_{\mathrm{T}}(1: P) \otimes \mathbf{S}_{\mathrm{r}, i, r} \otimes \mathbf{S}_{\mathrm{t}, i, r} .
$$

With the similar derivation to the previous $\mathrm{RD}$ algorithm, the rank of $\mathbf{U}_{r}$ is equal to the number of the distinct expressions of 


$$
\begin{aligned}
& e^{j 2 \pi \beta \mathbf{f}_{\mathrm{s}}(p-1)} \cdot\left(f_{q, 0}+\cdots+f_{q, Q-1} e^{j 2 \pi \mathbf{f}_{\mathrm{s}}(q-1)}\right) \\
& \cdot\left(g_{o, 0}+\cdots+g_{o, O-1} e^{j 2 \pi \gamma \mathbf{f}_{\mathrm{s}}(o-1)}\right),
\end{aligned}
$$

where $p=1, \ldots, P, q=1, \ldots, Q$, and $o=1, \ldots, O$.

Equation (25) is also the linear combination of (21). Thus, the clutter LDOF of this algorithm is also equivalent to the two previous algorithms.

3.4. Clutter LDOF for Beam-Space Post-Doppler (BS PostDop) $R D$ MIMO-STAP. In this case, the transformed spatial-temporal steering vector is

$$
\mathbf{S}_{i, r}=\mathbf{S}_{\mathrm{T}, i, r} \otimes \mathbf{S}_{\mathrm{r}, i, r} \otimes \mathbf{S}_{\mathrm{t}, i, r}
$$

Similarly, every row of simplified $\mathbf{U}_{r}$ has the form of

$$
\begin{aligned}
\left(h_{p, 0}+\cdots+h_{p, P-1} e^{j 2 \pi \beta \mathbf{f}_{s}(P-1)}\right) & \cdot\left(f_{q, 0}+\cdots+f_{q, Q-1} e^{j 2 \pi \mathbf{f}_{s}(q-1)}\right) \\
& \cdot\left(g_{o, 0}+\cdots+g_{o, O-1} e^{j 2 \pi \gamma \mathbf{f}_{s}(o-1)}\right),
\end{aligned}
$$

where $p=1, \ldots, P, q=1, \ldots, Q$, and $o=1, \ldots, O$.

Obviously, the rank of $\mathbf{U}_{r}$, that is, the clutter LDOF, is the number of distinct expressions of (21).

Therefore, the equivalence of LDOFs between different RD MIMO-STAP algorithms is proved.

\section{Estimation of Clutter LDOF under Different Conditions}

In this section, we develop the clutter LDOF estimation rule for airborne MIMO radar with different $\beta$ and $\gamma$. Due to the conclusion in Section 3, the generalized formula of clutter LDOF for one of the four reduced-dimension algorithms is only required to be given. In this section, we choose ES preDop RD algorithms to show how to get the clutter LDOF. According to the analysis of Section 3.1, we notice that the clutter LDOF is equivalent to the number of distinct elements in the set $\Theta=(q-1)+\beta(p-1)+\gamma(o-1)$, where $p=1, \ldots$, $P, q=1, \ldots, Q$, and $o=1, \ldots, O$. Therefore, what we only need to do is find out the number of distinct elements in $\Theta$.

4.1. Clutter $L D O F$ with $\beta \leq Q$. When $\beta \leq Q$, there are only $Q+\beta(P-1)$ different elements of set $\{(q-1)+\beta(p-1)$ $\left.\left.\right|_{q=1: Q, p=1: P}\right\}$. Thus, $\Theta$ can be rewritten as

$$
\Theta^{*}=\left\{l-1+\left.\gamma(o-1)\right|_{l=1: Q+\beta(P-1), o=1: O}\right\} .
$$

Now, the influence of $\gamma$ should be taken into account. If $\gamma \leq Q+\beta(P-1)$, with the same analysis, the number of different elements in $\Theta^{*}$, that is, the clutter LDOF, is

$$
k_{r}=Q+\beta(P-1)+\gamma(O-1)
$$

If $\gamma>Q+\beta(P-1), \Theta^{*}$ has no repeat element, and the clutter LDOF is thus given by

$$
k_{r}=(Q+\beta(P-1)) O \text {. }
$$

4.2. Clutter $L D O F$ with $\beta>Q$. In this subsection, four different situations for LDOF estimation are analyzed.

(1) $Q<\beta \leq Q+\gamma(O-1)$ and $\gamma \leq Q$.

Similar with the case $\beta \leq Q$ and $\gamma \leq Q+\beta(P-1)$, the clutter LDOF is given as

$$
k_{r}=Q+\beta(P-1)+\gamma(O-1) .
$$

(2) $Q<\beta \leq Q+\gamma(O-1)$ and $\gamma>Q+\beta(P-1)$.

In this case, the elements of set $\Theta$ are all different from each other, and the clutter LDOF is thus

$$
k_{r}=O P Q
$$

(3) $\beta>Q+\gamma(O-1)$ and $\gamma \leq Q$.

Corresponding to $\beta \leq Q$ and $\gamma>Q+\beta(P-1)$, the clutter LDOF is

$$
k_{r}=[Q+\gamma(O-1)] P .
$$

(4) $\beta>Q+\gamma(O-1)$ and $Q<\gamma \leq Q+\beta(P-1)$.

Parallel with situation 2, the clutter LDOF is equal to

$$
k_{r}=O P Q \text {. }
$$

\subsection{LDOF Estimation Rule Discussions}

Remark 1. The generalized estimation rule of clutter LDOF with $Q<\beta \leq Q+\gamma(O-1)$ and $Q<\gamma \leq Q+\beta(P-1)$ is not analyzed in this paper, which needs further study.

Remark 2. If $Q=N, P=K$, and $O=M$, which means that the dimension of clutter has not been reduced, the proposed estimation rule can be viewed as the generalized form of the extended Brennan's rule to the MIMO radar case [12], in which $\beta \leq N$ and $\gamma \leq N+\beta(K-1)$ are considered to be sufficient.

Remark 3. In the case of $M=1$, the MIMO radar will be degenerated to the SIMO radar case, and thus, our estimation rule is automatically reduced to the generalized form of clutter LDOF formulas proposed in [17], where the influence of $\beta$ is not necessary to be analyzed.

\section{Simulation Results}

In this section, simulations with different conditions are conducted to verify the effectiveness of the proposed clutter LDOF estimation rule and demonstrate its guidance to the design of RD STAP processor. First of all, we assume the following simulated parameters: side-looking airborne 8element monostatic MIMO array radar, whose wavelength 


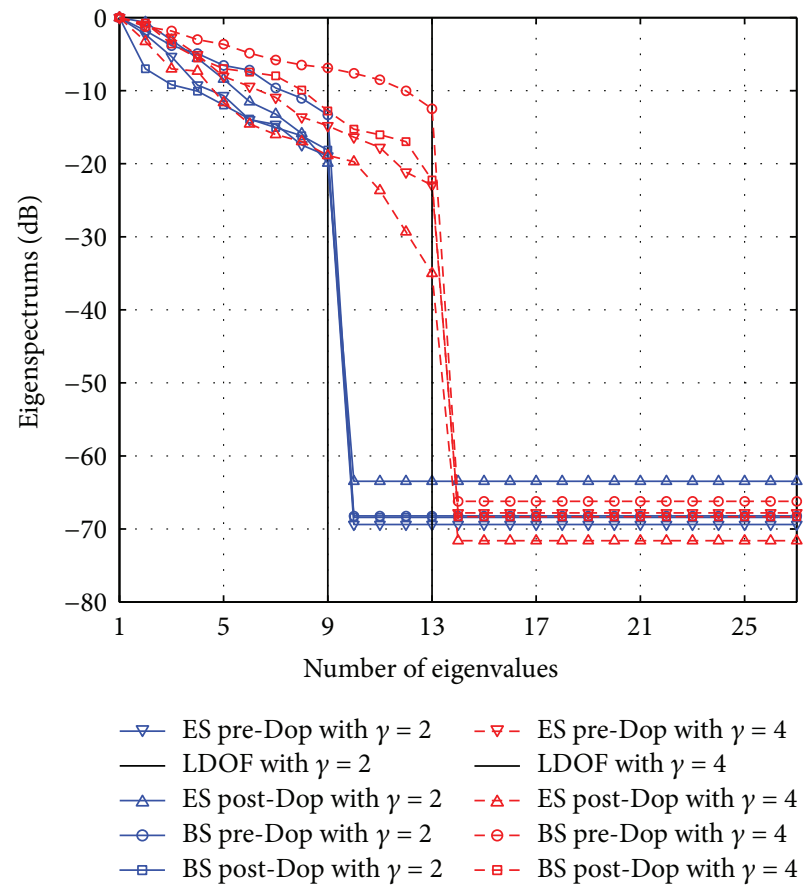

(a) Estimated results derived from (29)
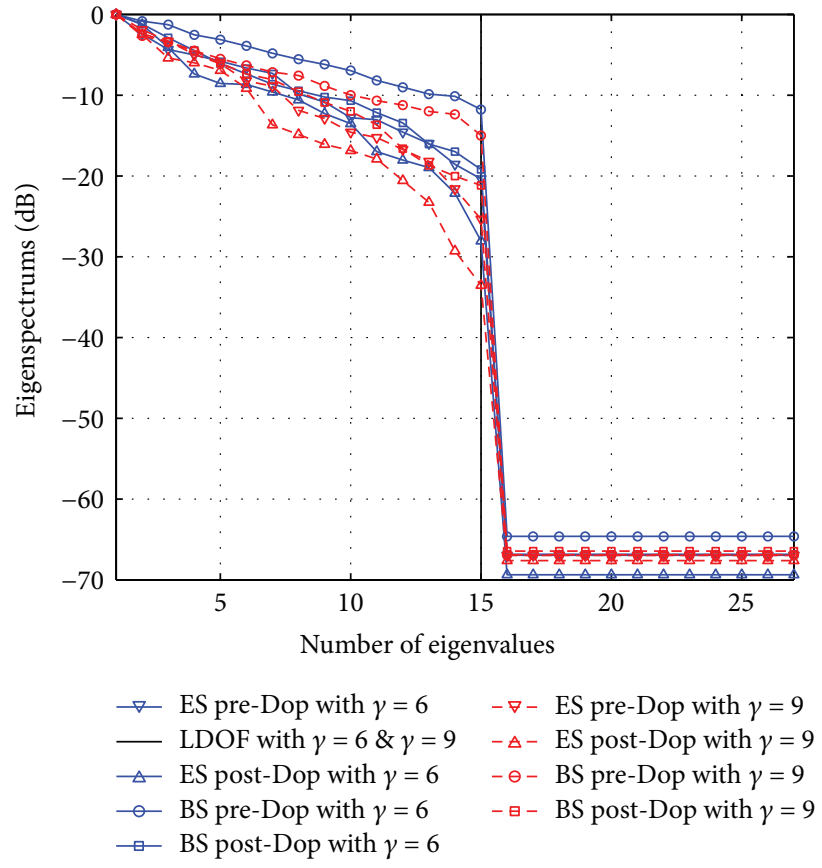

(b) Estimated results derived from (30)

FIGURE 2: Clutter eigenspectrums of RD MIMO-STAP algorithms to verify (29) and (30).

is $0.23 \mathrm{~m}$, is located at the altitude $6000 \mathrm{~m}$ with constant velocity $140 \mathrm{~m} / \mathrm{s}$; the receiving elements are uniformly spaced by $0.115 \mathrm{~m}$ and the transmitting elements are uniformly spaced by $d_{T}=0.115 \cdot \gamma ; \mathrm{CNR}=60 \mathrm{~dB}$; and the number of pulses is 8 .

For element-space RD algorithms, the first 3 receiving elements and 3 transmitting elements are selected; for beam-space $\mathrm{RD}$ algorithms, received beams with indices 1 , 3 , and 5 and transmitted beams with indices 3,5 , and 7 are selected, which means that $Q=O=3 . P=3$ denotes that the first 3 pulses are selected for pre-Doppler algorithms, and Doppler bins with indices 1 to 3 are selected for postDoppler algorithms. $P=4$ denotes that the first 4 pulses are selected for pre-Doppler algorithms, and Doppler bins with indices 1 to 4 are selected for post-Doppler algorithms. In all figures, the LDOFs estimated using the proposed rule is plotted by vertical solid black lines.

5.1. Effectiveness of the Proposed Estimation Rule. Firstly, $P=3$ is considered. The clutter eigenspectrums with $\beta=$ $1, \gamma=2$ and $\beta=1, \gamma=4$ are shown in Figure 2(a), which is used to verify the estimated results derived from (29). As is illustrated in Figure 2(a), the eigenspectrums exhibit sharp cutoffs along the calculated ranks, which indicates that (27) can give an accurate estimation of clutter LDOF. Figure 2(b) shows clutter eigenspectrums with $\beta=1, \gamma=6$ and $\beta=1, \gamma=9$ to verify the accuracy of (30). One can see from Figure 2(b) that the clutter LDOF does not change with the increasing $\gamma$, and the estimated LDOF is exactly the same as the eigenvalue decomposition method.
Secondly, $P=4$ is considered. The eigenspectrums with $\beta=4, \gamma=1$ and $\beta=5, \gamma=1$ are plotted in Figure 3(a), which is utilized to verify the effectiveness of (31). The eigenspectrums of clutter in Figure 3(b) are exploited to validate the accuracy of (33), where we set $\beta=7, \gamma=1$ and $\beta=8, \gamma=1$. From Figure 3(a), we can see that the numbers of eigenvalues are 17 and 20, which are exactly the same as the numbers calculated by (31). Then, we can observe from Figure 3(b) that the eigenspectrums display sharp cutoffs at the same position of estimated LDOF for different $\beta$, which indicates that the LDOF does not increase as $\beta$ goes up, so the LDOF could be accurately estimated by (33).

Finally, (32) with $P=3$ and (34) with $P=4$ are verified by Figures $4(\mathrm{a})$ and $4(\mathrm{~b})$, respectively. In Figure $4(\mathrm{a}), \beta=4, \gamma$ $=12$ and $\beta=4, \gamma=13$. In Figure $4(\mathrm{~b}), \beta=12, \gamma=4$ and $\beta=$ $13, \gamma=4$. We can observe that all eigenspectrums decrease gradually, which means that the clutter covariance matrix is nonsingular.

5.2. Guidance to the Dimension-Reduced STAP Processor Designing. To suppress clutter effectively, the DOF of RD STAP processors should be larger than that of the clutter. Thus, in this subsection, we demonstrate how to utilize the proposed LDOF estimation rule to guide the design of STAP processors. Without loss of generality, BS post-Dop RD algorithm is only considered. Assuming $\beta=4, \gamma=12, O=3, Q=3$, and $P=3$, the clutter LDOF obtained from (32) is 27 , which is equal to the DOF of system. In this case, the clutter will not be well suppressed. Therefore, with the instruction of (29), Q is changed to 5, 


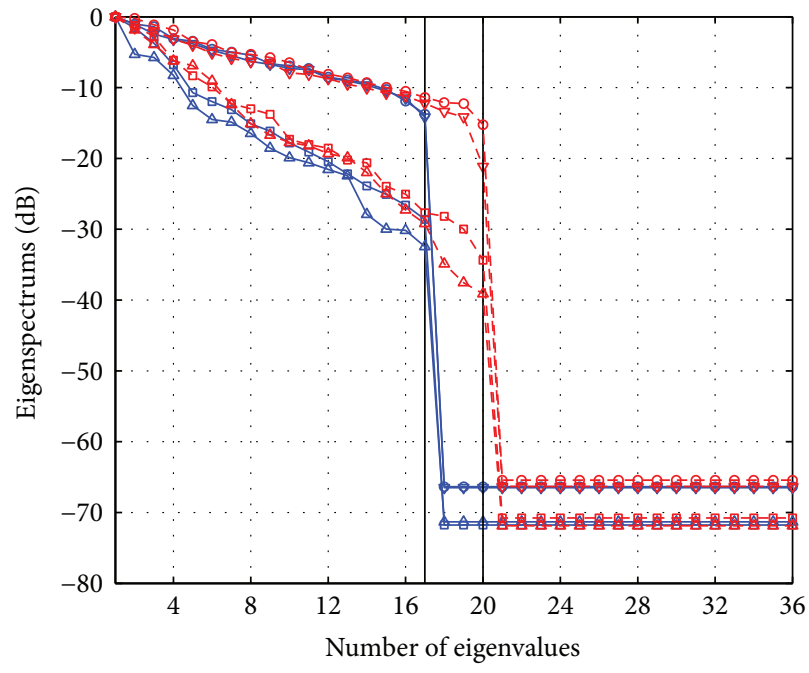

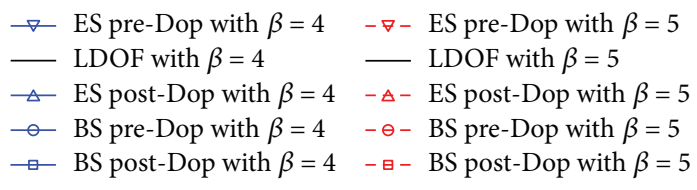

(a) Estimated results derived from (31)

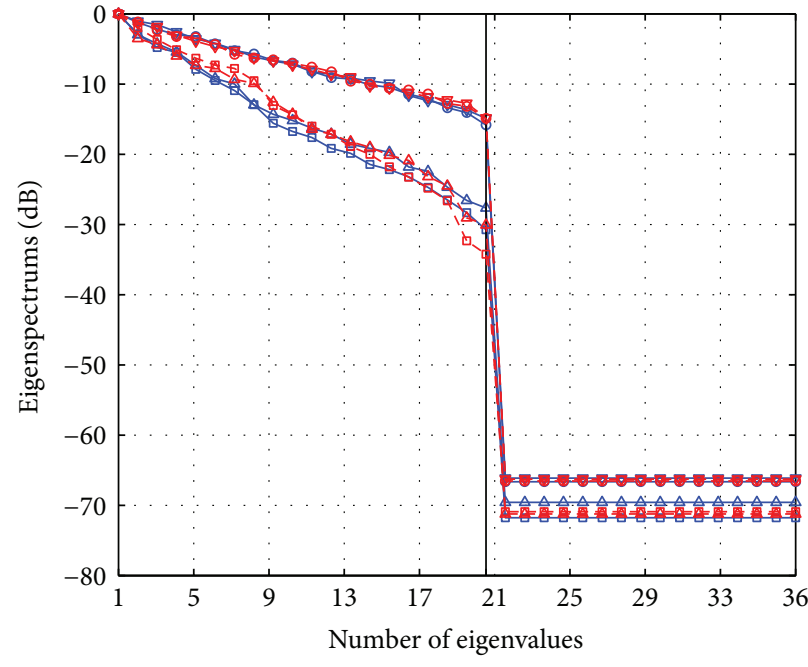

$\rightarrow$ ES pre-Dop with $\beta=7 \quad-\nabla-$ ES pre-Dop with $\beta=8$

— LDOF with $\beta=7 \quad$ LDOF with $\beta=7 \& \beta=8$

$\triangle$ ES post-Dop with $\beta=7 \quad-\Delta-$ ES post-Dop with $\beta=8$

$\rightarrow$ BS pre-Dop with $\beta=7 \quad-\theta-$ BS pre-Dop with $\beta=8$

$\rightarrow$ BS post-Dop with $\beta=7 \quad-\bullet-$ BS post-Dop with $\beta=8$

(b) Estimated results derived from (33)

Figure 3: Clutter eigenspectrums of RD MIMO-STAP algorithms to verify (31) and (33).
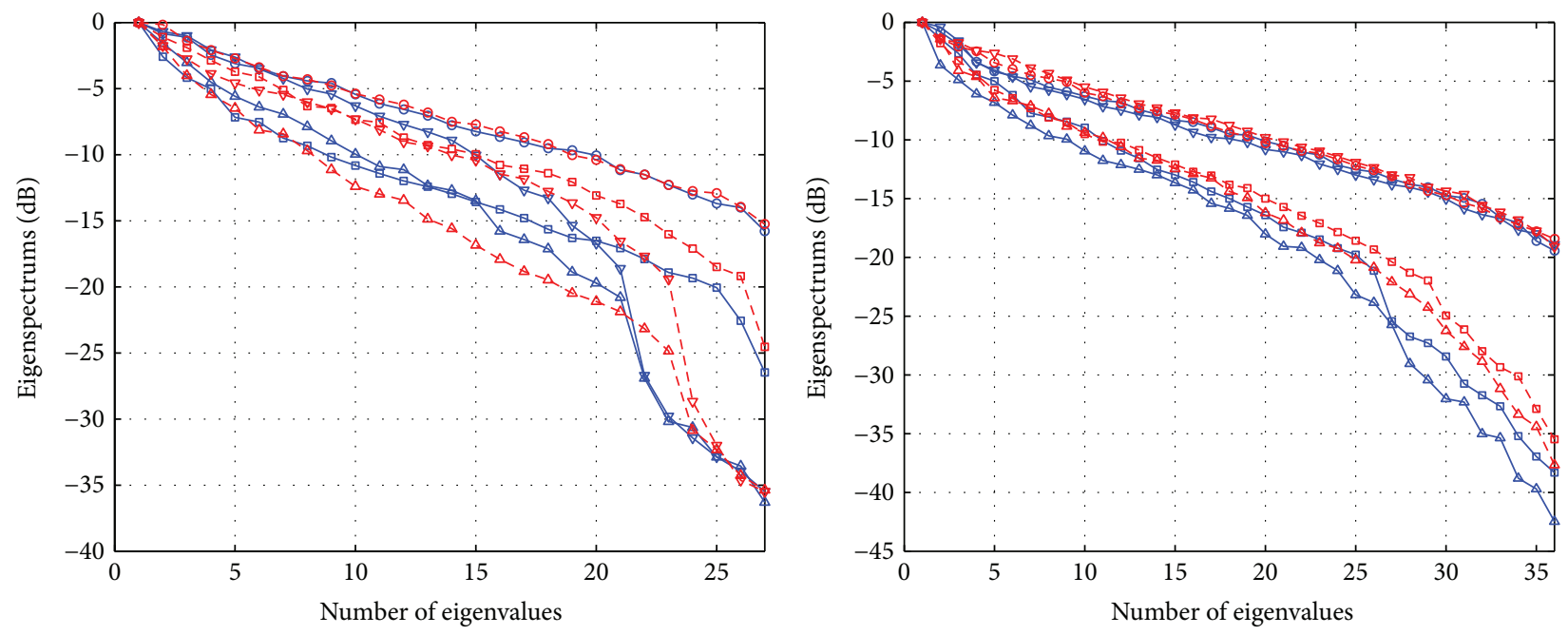

$\nabla$ ES pre-Dop with $\beta=4, \gamma=12$

LDOF with $\beta=4, \gamma=12$ \& $\gamma=13$

$\triangle$ ES post-Dop with $\beta=4, \gamma=12$

$\rightarrow$ BS pre-Dop with $\beta=4, \gamma=12$

$\rightarrow$ BS post-Dop with $\beta=4, \gamma=12$

$-\nabla$ - ES pre-Dop with $\beta=4, \gamma=13$

- $\triangle$ - ES post-Dop with $\beta=4, \gamma=13$

- $\theta$ - BS pre-Dop with $\beta=4, \gamma=13$

- - BS post-Dop with $\beta=4, \gamma=13$

(a) Estimated results derived from (32)

$\rightarrow$ ES pre-Dop with $\beta=4, \gamma=12$
- LDOF with $\beta=4, \gamma=12 \& \gamma=13$
$\triangle \quad$ ES post-Dop with $\beta=4, \gamma=12$
$-\quad$ BS pre-Dop with $\beta=4, \gamma=12$
$\square-$ BS post-Dop with $\beta=4, \gamma=12$
$-\nabla-$ ES pre-Dop with $\beta=4, \gamma=13$
$-\triangle-$ ES post-Dop with $\beta=4, \gamma=13$
$-\ominus-$ BS pre-Dop with $\beta=4, \gamma=13$
$-\bullet-$ BS post-Dop with $\beta=4, \gamma=13$

(b) Estimated results derived from (34)

FIGURE 4: Clutter eigenspectrums of RD MIMO-STAP algorithms to verify (32) and (34).

then the clutter LDOF is 37, which is smaller than the DOF of the system. Furthermore, with the instruction of (30), $Q$ and $P$ are changed to 5 and 2, respectively, and the clutter LDOF is 27; it is also smaller than the DOF of the system. Thus, the clutter could be suppressed effectively. The above analysis can be confirmed by 


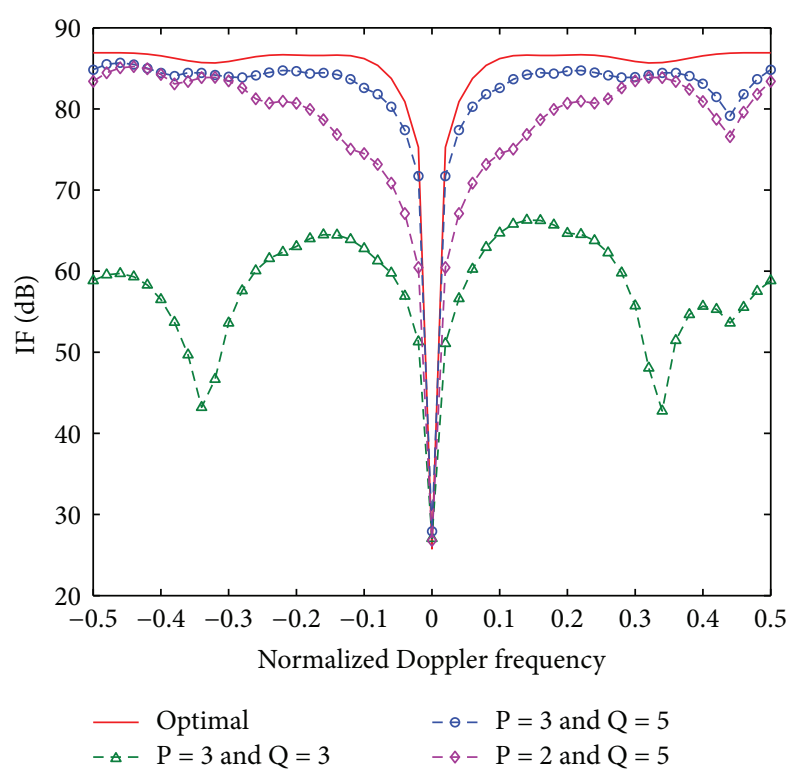

FIGURE 5: Improvement factor against target Doppler frequency for different RD MIMO-STAP processors.

improvement factor (IF) against the target-normalized Doppler frequency presented in Figure 5, where IF that illustrates the clutter suppression performance is defined as the ratio of output signal-clutter-noise ratio (SCNR) to the input SCNR.

\section{Conclusions}

In this paper, a generalized rule to estimate the clutter LDOF is proposed for MIMO radar. Four reduceddimension MIMO-STAP algorithms are introduced, and then, the LDOF equivalence of the four algorithms is proved. Finally, the proposed rule is considered with different $\gamma$ and $\beta$. The simulated results indicate the accuracy of the proposed rule. With the instruction of the proposed rule, the minimal DOF requirement could be determined and more appropriate system parameters can be chosen. Therefore, better clutter suppression performance of RD MIMO-STAP algorithms could be achieved. The estimation rule presented in this paper enjoys a good potential to guide the practical design of MIMOSTAP processors.

\section{Conflicts of Interest}

The authors declare no conflict of interest.

\section{Authors' Contributions}

All authors contributed extensively to the study presented in this manuscript. Yiduo Guo designed the main idea, methods, and experiments, carried out the experiments, and wrote the paper. Jian Gong supervised the main idea and edited the manuscript. Yu Xiao interpreted the results and provided many valuable suggestions to this study.

\section{Acknowledgments}

This work was supported in part by the National Natural Science Foundation of China under Grant nos. 61501501 and 61601502, the China Postdoctoral Science Foundation under Grant no. 2017M613075, and the Aeronautical Science Foundation of China under Grant no. 20160196003.

\section{References}

[1] L. E. Brennan and L. S. Reed, "Theory of adaptive radar," IEEE Transactions on Aerospace and Electronic Systems, vol. 9, no. 2, pp. 237-252, 1973.

[2] J. R. Guerci, Space-Time Adaptive Processing for Radar, Artech House, Norwood, MA, USA, 2003.

[3] E. Fishler, A. Haimovich, and R. Blum, "MIMO radar: an idea whose time has come," in Proceedings of the 2004 IEEE Radar Conference (IEEE Cat. No.04CH37509), pp. 71-78, Philadelphia, PA, USA, April 2004.

[4] J.-W. Xu, S.-Q. Zhu, and G.-S. Liao, "Space-time-range adaptive processing for airborne radar systems," IEEE Sensors Journal, vol. 15, no. 3, pp. 1602-1610, 2015.

[5] J. He, D.-Z. Feng, and L. Ma, "Reduced-dimension clutter suppression method for airborne multiple-input multipleoutput radar based on three iterations," IET Radar, Sonar and Navigation, vol. 9, no. 3, pp. 249-254, 2015.

[6] L. E. Brennan and F. M. Staudaher, "Subclutter visibility demonstration," Tech. Rep. RL-TR-92-91, Adaptive Sensors Inc., Santa Monica, CA, USA, 1992.

[7] J. Ward, "Space-time adaptive processing for airborne radar," Tech. Rep. 1015, MIT Lincoln Laboratory, Lexington, MA, USA, 1994.

[8] Q. Zhang and W. B. Mikhael, "Estimation of the clutter rank in the case of subarraying for space-time adaptive processing," Electronics Letters, vol. 33, no. 5, pp. 419-420, 1997.

[9] N. A. Goodman and J. M. Stiles, "On clutter rank observed by arbitrary arrays," IEEE Transactions on Signal Processing, vol. 55, no. 1, pp. 178-186, 2007.

[10] V. Varadarajan and J. L. Krolik, "Joint space-time interpolation for distorted linear and bistatic array geometries," IEEE Transactions on Signal Processing, vol. 54, no. 3, pp. 848-860, 2006.

[11] Y. Wu, J. Tang, and Y. Peng, "Clutter rank of multidimensional sparse array radar," in 2007 IEEE Radar Conference, pp. 463-468, Boston, MA, USA, April 2007.

[12] C.-Y. Chen and P. P. Vaidyanathan, "MIMO radar space-time adaptive processing using prolate spheroidal wave functions," IEEE Transactions on Signal Processing, vol. 56, no. 2, pp. 623-635, 2008.

[13] W. Xie, X. Zhang, Y. Wang, and Y. Zhu, "Estimation of clutter degrees of freedom for airborne multiple-input multipleoutput-phased array radar," IET Radar, Sonar and Navigation, vol. 7, no. 6, pp. 652-657, 2013.

[14] R. Li, J. Li, C. Luo, C. Han, and H. Li, "Estimation of clutter rank of wideband airborne MIMO radar," Electronics Letters, vol. 50, no. 25, pp. 1979-1980, 2014.

[15] X. Li, D. Feng, H.-W. Liu, and D. Luo, "Dimension-reduced space-time adaptive clutter suppression algorithm based on lower-rank approximation to weight matrix in airborne radar," IEEE Transactions on Aerospace and Electronic Systems, vol. 50, no. 1, pp. 53-69, 2014. 
[16] J. S. Goldstein and I. S. Reed, "Theory of partially adaptive radar," IEEE Transactions on Aerospace and Electronic Systems, vol. 33, no. 4, pp. 1309-1325, 1997.

[17] Z.-H. Zhang, W.-C. Xie, W.-D. Hu, and W.-X. Yu, "Local degrees of freedom of airborne radar clutter for STAP," IEEE Geoscience and Remote Sensing Letters, vol. 6, no. 1, pp. 97-101, 2009.

[18] Z.-H. Zhang, J.-B. Zhu, and Y.-L. Wang, "Local degrees of freedom of clutter for airborne space-time adaptive processing radar with subarrays," IET Radar, Sonar and Navigation, vol. 6, no. 3, pp. 130-136, 2012. 


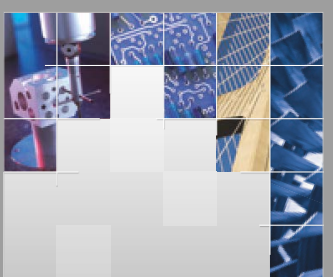

\section{Enfincering}
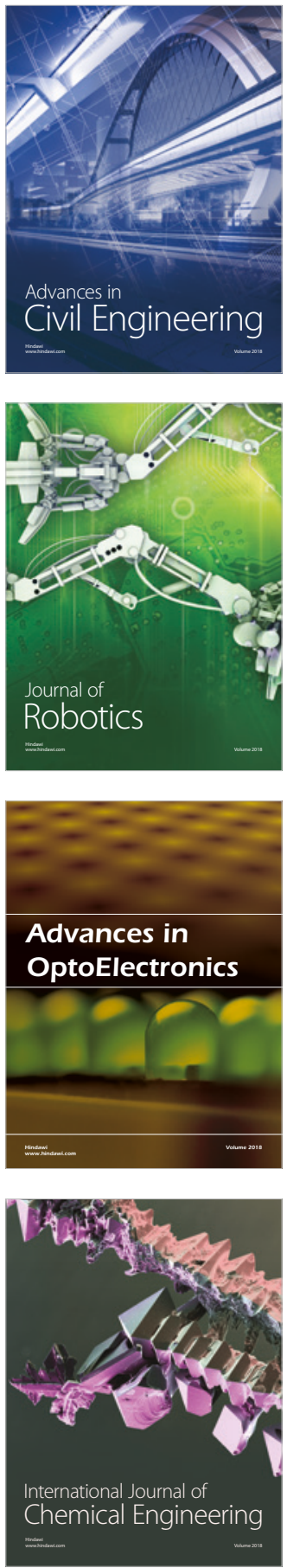

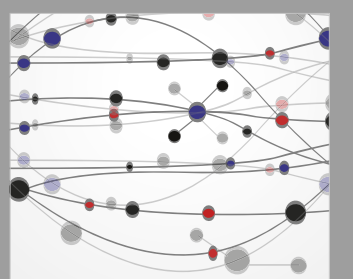

\section{Rotating \\ Machinery}

The Scientific World Journal

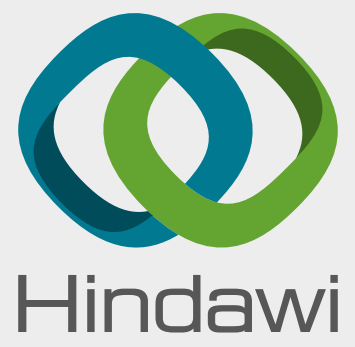

Submit your manuscripts at

www.hindawi.com
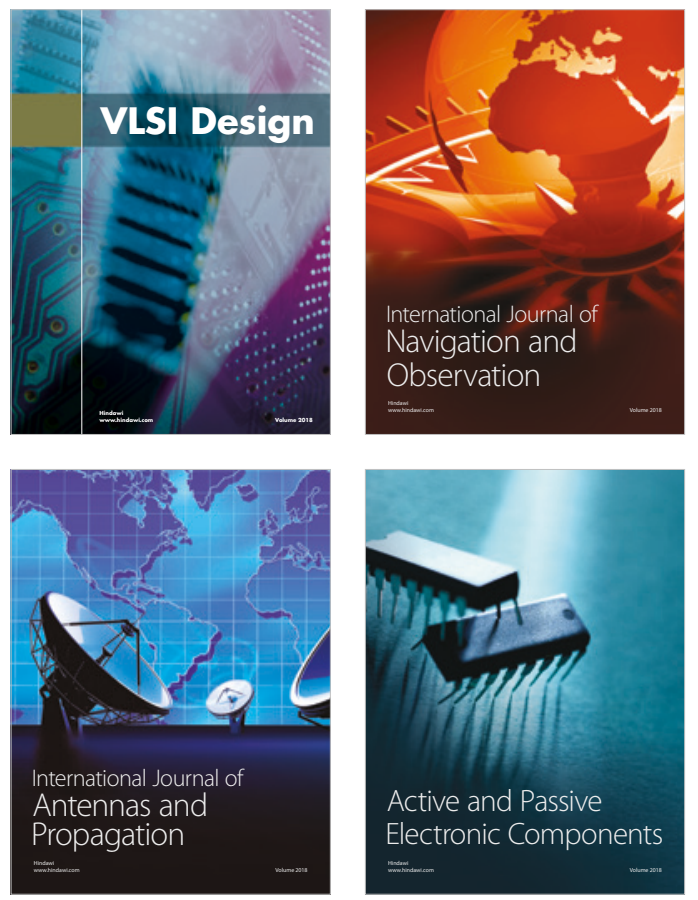
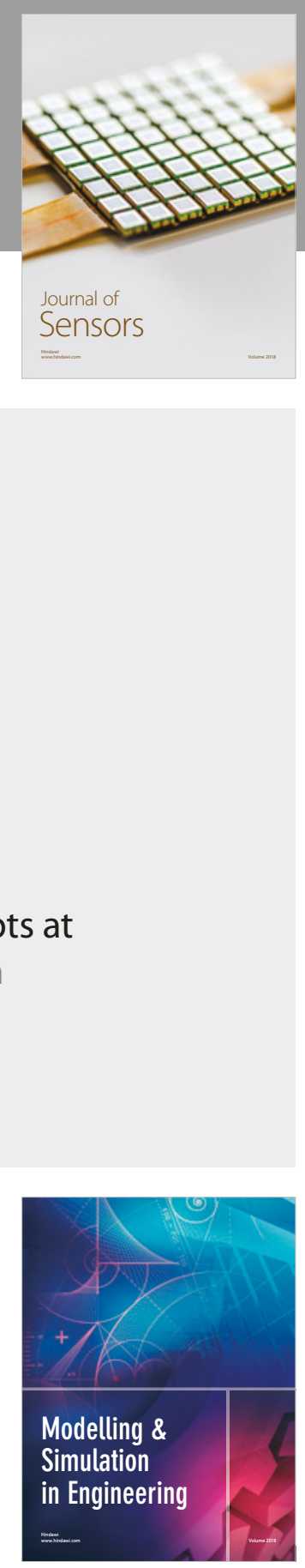

\section{Advances \\ Multimedia}
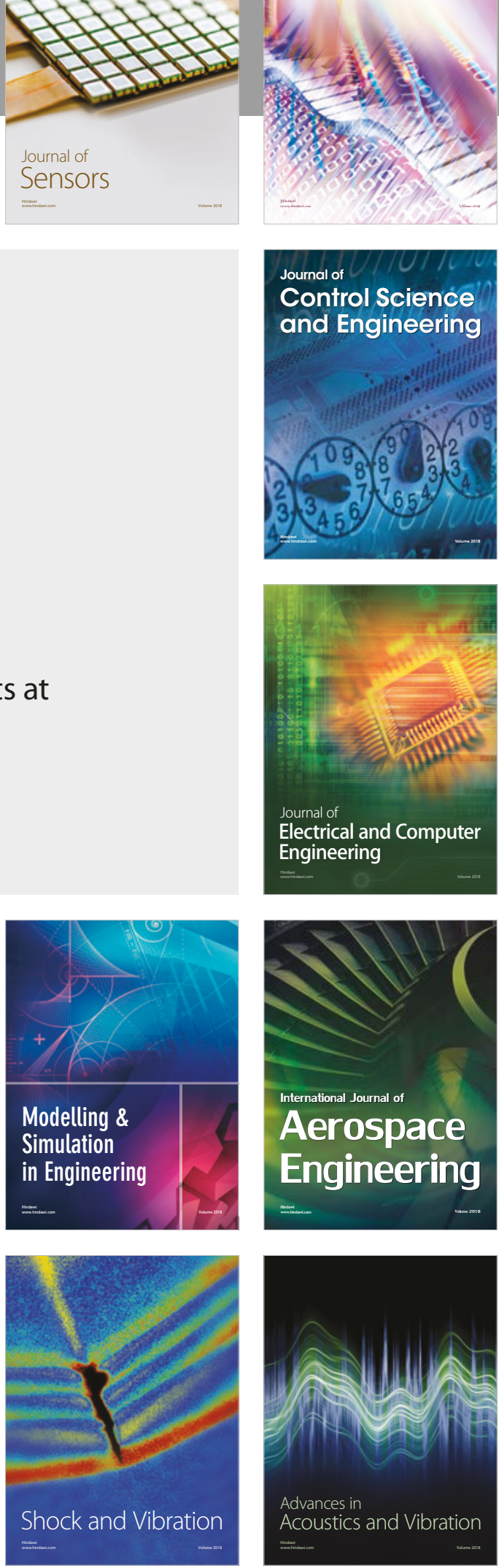